in vivo $35: 2197-2205(2021)$

doi:10.21873/invivo.12491

\title{
Finite Element Analysis of an Improved Correction System for Spinal Deformity
}

\author{
ZUBIN HE ${ }^{1 *}$, MEICHAO ZHANG ${ }^{2 *}$, WEIJIAN LI $^{3}$, ZHENGXUE LONG $^{1}$, \\ LINGPENG WANG ${ }^{4}$, QINGDI QUENTIN LI $^{5}$ and XIAOSHENG LU ${ }^{1}$ \\ ${ }^{1}$ People's Hospital of Baise, Baise, P.R. China; \\ ${ }^{2}$ Department of Anatomy, Southern Medical University, Guangzhou, P.R. China; \\ ${ }^{3}$ First People's Hospital of Yongkang City, Yongkang, P.R. China; \\ ${ }^{4}$ Youjiang Medical University for Nationalities, Baise, P.R. China; \\ ${ }^{5}$ National Institutes of Health, Bethesda, MD, U.S.A.
}

\begin{abstract}
Background/Aim: Surgical treatment for spinal deformity aims to correct malformation, release the nerves, and reconstruct spinal stability. To explore and develop a new improved spinal correction system (ISCS) for clinical application, we studied the stability and biomechanical characteristics of the ISCS through finite element analysis and comparison of the ISCS with the pedicle screw and rod system (PSRS). Patients and Methods: Using L1-L3 CT image data of a normal adult male lumbar spine for establishment of L1-L3 finite element model, we established posterior internal fixation models for a comparative finite element analysis of PSRS and ISCS. An axial load of $500 \mathrm{~N}$ and a moment of $10 \mathrm{~N} \cdot \mathrm{m}$ were applied to $\mathrm{L} 1$ to simulate flexion, extension, lateral bending, and axial rotation. Stress distribution characteristics, load sharing, strain bending stiffness and strain angle change of the models were measured. Results: In flection and extension directions, the maximum stress of the L2 vertebral body and the L1/2 and L2/3 discs in PSRS was less than that of ISCS. In lateral bending and axial rotation directions, the maximum stress between PSRS and ISCS was similar. However, the stress shielding rate of $L 2, L 1 / 2$, and $L 2 / 3$ intervertebral discs in ISCS was significantly lower than that of PSRS. We also
\end{abstract}

This article is freely accessible online.

*These Authors contributed equally to this study.

Correspondence to: Dr. Xiaosheng Lu, People's Hospital of BaiSe, 98 Urban and Rural Road, Youjiang District, BaiSe 533000, P.R. China. Tel: +86 13481697043, Fax: 07762840980, e-mail: luxiaosheng05@126.com

Key Words: Improved spine correction system (ISCS), pedicle screw and rod system (PSRS), finite element analysis, biomechanics, spinal deformity, scoliosis. found that both models had similar angular displacement and maximum displacement in lateral bending direction, but PSRS had a lower angular displacement and maximum displacement in flection and extension directions. Finally, we showed that PSRS had similar angular displacement and a lower maximum displacement compared with ISCS in axial rotation, whereas ISCS had lower bending stiffness than PSRS in different directions. Conclusion: ISCS can effectively fix spinal deformities compared to PSRS. ISCS provides a new option for orthopedic surgery treatment of scoliosis and, therefore, warrants further clinical studies in patients with other spinal deformities.

Surgical operation for spinal deformity such as congenital scoliosis, adolescent scoliosis, and idiopathic scoliosis is designed to correct malformation, release the nerves, and reconstruct spinal stability. The pedicle screw and rod system (PSRS) is able to achieve strong stability with screws crossing spinal three columns, and it has been widely accepted as the gold standard for the treatment of scoliosis. However, there are inherent weaknesses in PSRS. For instance, high risk of nailing and high technical requirements are demanded for PSRS. In addition, the stress shielding effect due to rigid fixing would reduce the stress stimulus and bone mass, while stress concentration may result in breakage of screw or rod. Moreover, adjacent vertebral disease is another hot issue with PSRS. In view of the above problems, a new concept of dynamic spinal internal fixation has been proposed. Recently, we designed a new improved spinal correction system (ISCS) (1) based on the Corhet à Appui Bilatéral (CAB) system invented by Csernátony et al. (2-4). The advantages of ISCS over PSRS are that its surgical operations are simple, convenient, and safe, because only transverse processes or lamina in ISCS need to be exposed. The transverse hook and multi-directional device in ISCS retain functional spinal motion under the premise of 
ensuring effective fixation and reducing stress shielding. In the present study, we conducted a finite element analysis of ISCS and evaluated its biomechanical characteristics in order to develop clinical application of ISCS as a new therapeutic option in patients with scoliosis and other spinal deformities.

\section{Patients and Methods}

Study subject. An adult male volunteer 40 years old, $170 \mathrm{~cm}$ in height and $60 \mathrm{~kg}$ in weight, was selected, and other spinal diseases were excluded by digital radiography (DR) and magnetic resonance imaging (MRI). Computerized tomography (CT) thin-section scan data of L1-3 were collected by light speed CT whose scanning conditions were $1 \mathrm{~mm}$ thick, $125 \mathrm{~mA}, 120 \mathrm{KV}$, and saved in digital imaging and communications of medicine (DICOM) format.

This study was undertaken with the permission of the local authority and the Medical Ethics Committee of the People's Hospital of BaiSe. All experiments were performed in accordance with the ethical standards of the 1995 Declaration of Helsinki, as revised in Tokyo (2004). Written informed consent and a comprehensive questionnaire were obtained from the study subject.

Establishment of the finite element analysis model of L1-3. CT data of DICOM format were imported into Mimics 19.0 and bone tissue was extracted by threshold segmentation and regional growth, and then smoothed by Geomagic-Freeform. Model was imported into ANSYS 15.0 after smoothing, polishing, and denoising by Geomagic Studio11.0. Intervertebral discs including nucleus pulposus and annulus fibrosus were created by three-dimensional reconstruction and Boolean subtraction. The annulus fibrosus ligament, intertransverse ligament, yellow ligament, interspinous ligament, superior spinous ligament, and anterior and posterior longitudinal ligaments were added manually that simulated by bean element with only proper tension and no pressure. Cortical bone, cancellous bone, and intervertebral disc were all constituted by solid 187 tetrahedral unit. Capsular ligament enclosing the facet joints was defined as aspect contact without frictional characteristic. There were 86,231 tetrahedron elements, 4,749 rope units, and 1,288 contact units in the model including 12 material properties and three-unit types with a total of 28,994 nodes. Setting the modulus of elasticity, Poisson's ratio, and cross-sectional area (Table I) for bone, cartilage, ligaments, and intervertebral discs of the model (Figure 1) according to the literature (5-7).

Verifying the validity of the finite element model of L1-3. The validity of finite element model of L1-3 was verified by mean stiffness value. Mean stiffness formula: $K=M / \theta$ ( $\mathrm{M}=$ moment, $\theta=$ angular rotation). A moment of $10 \mathrm{~N} \bullet \mathrm{m}$ was applied to the model for calculating the mean stiffness in the directions of flexion, extension, lateral bending, and axial
Table I. Material properties of each structure in the finite element analysis models.

\begin{tabular}{lccc}
\hline Materials & $\begin{array}{c}\text { Elastic } \\
\text { modulus/MPa }\end{array}$ & $\begin{array}{c}\text { Poisson's } \\
\text { ration }\end{array}$ & $\begin{array}{c}\text { Cross section } \\
\text { area/mm }\end{array}$ \\
\hline Cortical bone & $12,000.0$ & 0.300 & - \\
Cancellous bone & 100.0 & 0.200 & - \\
Ligamentum flavum & 19.5 & 15 & 40.0 \\
Intertransverse ligament & 58.7 & 32 & 1.8 \\
Interspinous ligament & 11.6 & 13 & 40.0 \\
Supraspinal ligament & 15.0 & 22 & 30.0 \\
Nucleus pulposus & 1.0 & 0.499 & - \\
Annulus fibrosus & 4.2 & 0.450 & - \\
Anterior longitudinal & 20.0 & 20 & 63.7 \\
ligament & 20.0 & 12 & 20.0 \\
Posterior longitudinal & & & \\
ligament & 61,000 & 0.33 & - \\
Memory alloy & 110,000 & 0.25 & - \\
Titanium alloy & & & \\
\hline
\end{tabular}

rotation. Measured values were compared with literature (89) for verification of its validity.

Establishment of the finite element analysis models of PSRS and ISCS. For establishing the finite element model of L1-3 with PSRS, the model was composed of tetrahedral solid units. Interface among spine, screw and rod set as ideal gluing, and the degrees of freedom in all directions were limited. To simulate the posterior PSRS operation, screws were implanted into bilateral pedicles, rods with physiological curvature of lumbar spine, and all the nuts tightened at last (Figure 1A and B). For establishing the finite element model of L1-3 with ISCS. The ISCS was mainly made of medically used titanium alloy. Transverse process hooks and rods were made of memory alloy without magnetism. To simulate the posterior ISCS operation, the transverse process hooks were shaped and implanted into bilateral transverse process after cooling and grasped the transverse process after thawing. Implanting the rod into the U-shaped groove of the multi-directional device and tightening all the nuts (Figure $1 \mathrm{C}$ and D).

The stress tests and evaluation index. The stress loading is defined as the degree of freedom of L3 lower border. An axial load of $500 \mathrm{~N}$ and a moment of $10 \mathrm{~N} \bullet \mathrm{m}$ were applied to L1 superior border to simulate motion in the directions of flexion, extension, lateral bending, and axial rotation. Data extraction: (i) Under the same loading condition, the maximum stress of the two intervertebral discs (L1/2 and L2/3) and three centrums (L1, L2, L3) from the three models (normal model, PSRS, ISCS) were measured. (ii) Under the same loading condition, the maximum displacement, angular strain, bending stiffness, and stress shielding of the three models (normal model, PSRS, ISCS) were calculated. 


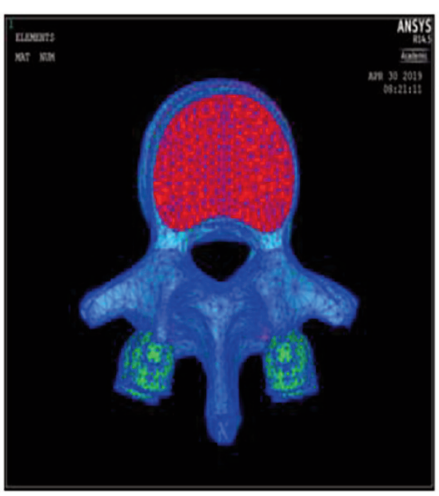

(A) Model PSRS

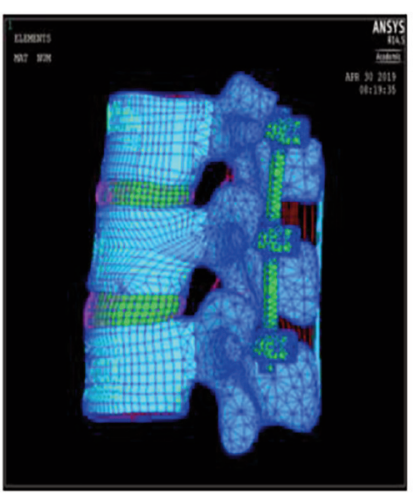

(B) Model PSRS

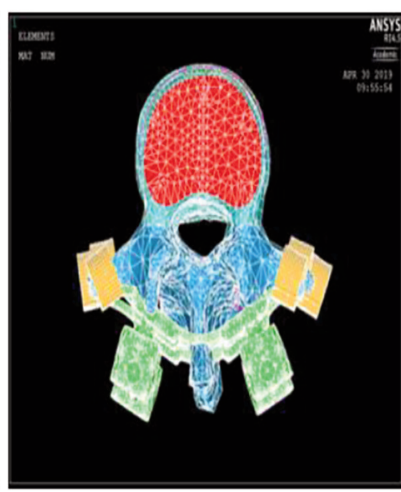

(C) Model ISCS

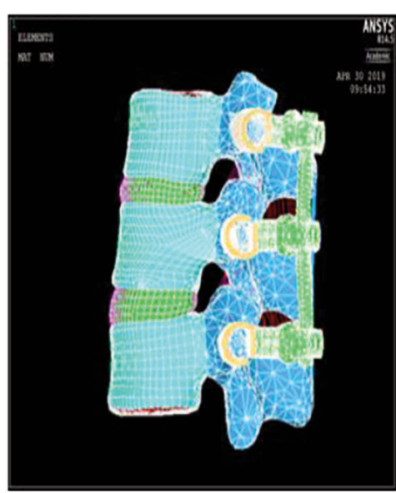

(D) Model ISCS

Figure 1. Finite element models. ISCS, Improved spinal correction system; PSRS, pedicle screw-rod system.

Table II. Verifying the stiffness of the normal model by finite element analysis.

\begin{tabular}{|c|c|c|c|c|c|c|c|c|c|}
\hline & \multicolumn{3}{|c|}{ Flexion } & \multicolumn{3}{|c|}{ Extension } & \multicolumn{3}{|c|}{ Lateral bending } \\
\hline & Model & {$[8]$} & [9] & Model & {$[8]$} & [9] & Model & {$[8]$} & [9] \\
\hline $\operatorname{ROM}\left({ }^{0}\right)$ & 7.6 & 6.3 & 9.6 & 4.2 & 5.0 & 6.1 & 7.8 & 6.2 & 8.3 \\
\hline Stiffness $\left[\mathrm{N} \bullet \mathrm{m} /\left({ }^{0}\right)\right]$ & 1.32 & 1.59 & 1.04 & 1.38 & 2.0 & 1.64 & 1.28 & 1.61 & 1.20 \\
\hline
\end{tabular}

ROM, Range of motion.

\section{Results}

Testing the validity of the finite element analysis models. According to the literature (8-9), the same load was applied to the models and measured values are shown in Table II. The range of motion (ROM) of the models in flexion and lateral bending was between the reference value. The ROM of the models in extension was lower than the reference value. In view of the difference in initial states during CT scanning, the models were considered to be effective.

The maximum stress of the intervertebral discs in different directions. As shown in Table III and Figure 2, during flexion and extension, the maximum stress of L1/2 and L2/3 discs in the model for PSRS was less than the model for ISCS, and the extension was more obvious. The maximum stress of L1/2 and L2/3 in lateral bending was close to that in rotation, and it was slightly larger in the model for PSRS than the model for ISCS (Figure 2).

The maximum stress of the centrum in different directions. As seen in Table IV and Figure 3, the L1 centrum in the model for PSRS had greater stress than the model for ISCS in axial compression, flexion, extension, lateral bending, and axial rotation, whereas the L2 centrum in the model for
Table III. $500 \mathrm{~N}$ axial load and $10 \mathrm{~N} \bullet \mathrm{m}$ moment of force bend forward, backward extension, left bending, and left rotation. The stress (MPa) of L1/2 and L2/3 intervertebral discs of the three models, normal model, PSRS, and ISCS.

\begin{tabular}{|c|c|c|c|c|c|c|}
\hline \multirow[b]{2}{*}{ Loaded state } & \multicolumn{2}{|c|}{ Normal model } & \multicolumn{2}{|c|}{ PSRS } & \multicolumn{2}{|c|}{ ISCS } \\
\hline & $\mathrm{L} 1 / 2$ & $\mathrm{~L} 2 / 3$ & $\mathrm{~L} 1 / 2$ & $\mathrm{~L} 2 / 3$ & $\mathrm{~L} 1 / 2$ & $\mathrm{~L} 2 / 3$ \\
\hline Vertical road & 0.52 & 0.61 & 0.57 & 0.69 & 0.52 & 0.59 \\
\hline Flexion & 1.28 & 1.11 & 0.79 & 0.80 & 1.11 & 0.90 \\
\hline Extension & 1.73 & 1.63 & 0.56 & 0.58 & 1.14 & 1.04 \\
\hline Left lateral bending & 1.63 & 1.79 & 0.83 & 0.94 & 0.73 & 0.82 \\
\hline Left rotation & 1.79 & 1.63 & 0.64 & 0.83 & 0.61 & 0.73 \\
\hline
\end{tabular}

ISCS, Improved spinal correction system; PSRS, pedicle screw-rod system.

PSRS had lower stress than the model for ISCS in flexion, extension, and axial rotation, but it was similar in lateral bending between the two models.

The stress shielding rate of the intervertebral disc and L2 centrum in different directions. As shown in Table $\mathrm{V}$, the disc L1/2, disc L2/3, and centrum L2 had lower stress shielding rate in the model for ISCS than the model for 


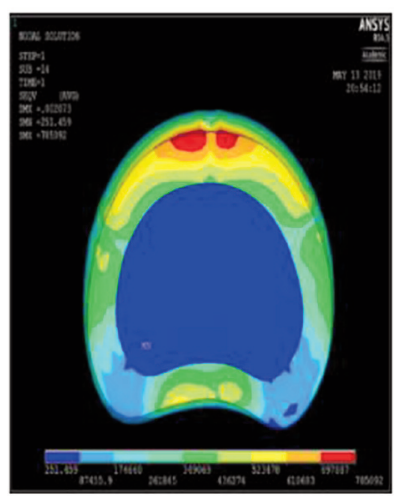

L12 of PSRS in flexion

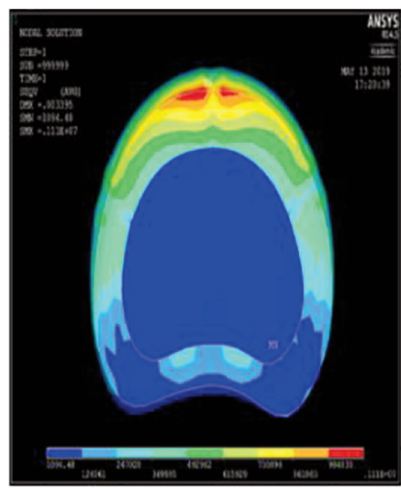

L12 of ISCS in flexion

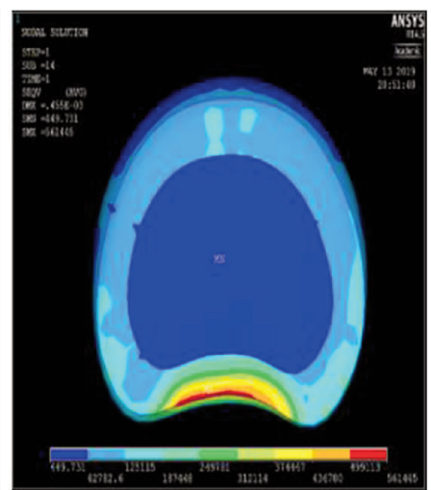

L12 of PSRS in extension

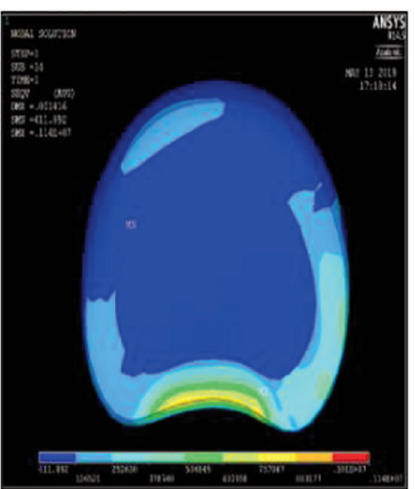

L12 of ISCS in extension

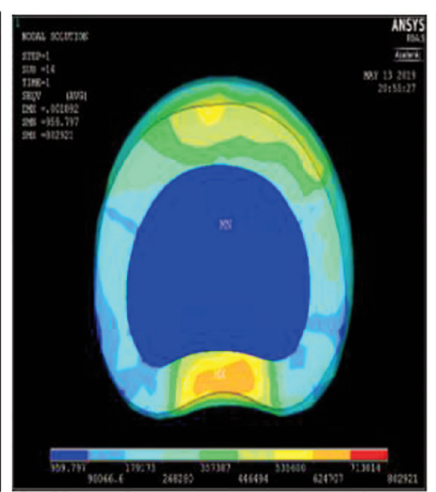

L23 of PSRS in flexion

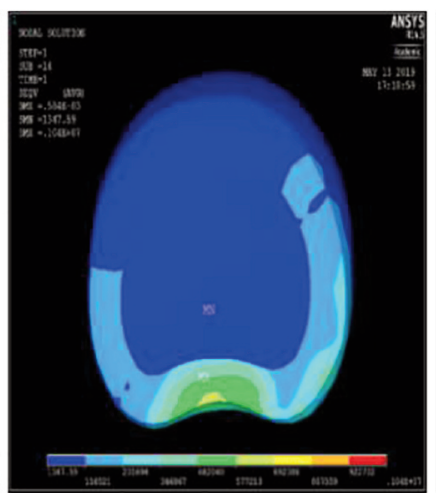

L23 of ISCS in flexion

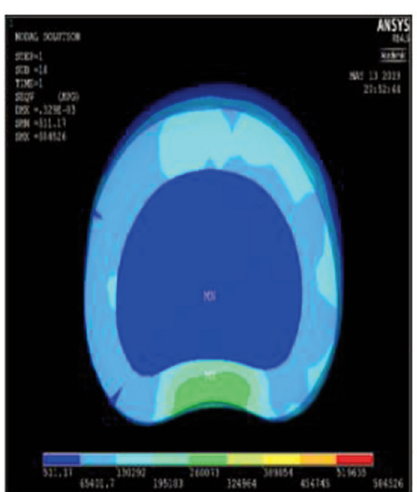

L23 of PSRS in extension

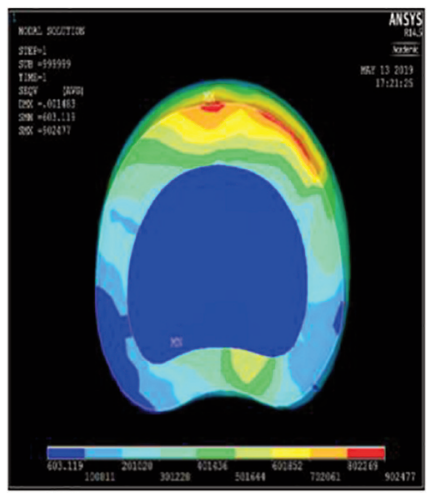

L23 of ISCS in extension

Figure 2. The maximum stress of the intervertebral disc during flexion and extension. ISCS, Improved spinal correction system; PSRS, pedicle screwrod system.

Table IV. The maximum stress of each vertebral body of the three models under the same load condition in flexion, extension, left bending, and left rotation.

\begin{tabular}{|c|c|c|c|c|c|c|c|c|c|}
\hline \multirow[b]{2}{*}{ Loaded state } & \multicolumn{3}{|c|}{ Normal model } & \multicolumn{3}{|c|}{ PSRS } & \multicolumn{3}{|c|}{ ISCS } \\
\hline & $\mathrm{L} 1$ & L2 & L3 & L1 & $\mathrm{L} 2$ & L3 & L1 & $\mathrm{L} 2$ & L3 \\
\hline Vertical road & 12.9 & 7.75 & 12.7 & 11.3 & 6.82 & 7.78 & 12.9 & 26.8 & 12.6 \\
\hline Flexion & 34.7 & 14.4 & 17.0 & 33.4 & 10.0 & 18.4 & 33.7 & 12.4 & 20.8 \\
\hline Extension & 20.8 & 21.8 & 18.4 & 33.1 & 4.34 & 18.5 & 26.1 & 9.01 & 15.6 \\
\hline Left lateral bending & 23.2 & 20.8 & 16.0 & 30.9 & 9.43 & 29.3 & 27.9 & 9.86 & 23.7 \\
\hline Left rotation & 14.5 & 12.2 & 14.9 & 15.2 & 9.68 & 9.00 & 14.8 & 10.6 & 14.6 \\
\hline
\end{tabular}

ISCS, Improved spinal correction system; PSRS, pedicle screw-rod system.

PSRS in flexion and extension. While in lateral bending, the disc L1/2 and L2/3 had slightly higher stress shielding rate in the model for PSRS than the model for ISCS, but it is similar in the centrum L2 between the two models. However, in axial rotation, the stress shielding rate of the centrum L2 was lower in the model for ISCS, and the stress shielding rate of discs was similar between the two internal fixation models.
The angular displacement and bending stiffness in different directions. As seen in Table VI, the model for PSRS had higher bending stiffness and smaller angular displacement than the model for ISCS, which was obvious in sagittal plane.

The maximum placement of $L 1$ in different directions. As shown in Table VII and Figure 4, the maximum placement of L1 was longer in the model for ISCS than the model for 


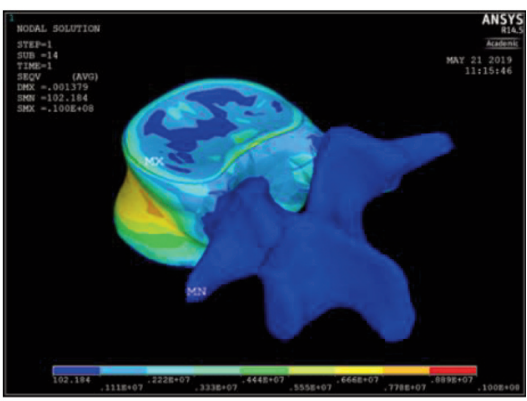

L2 of PSRS in flexion

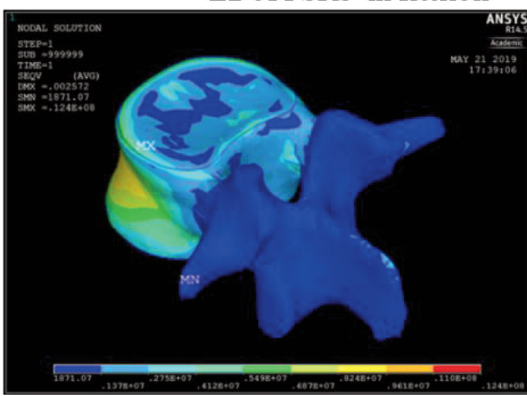

L2 of ISCS in flexion
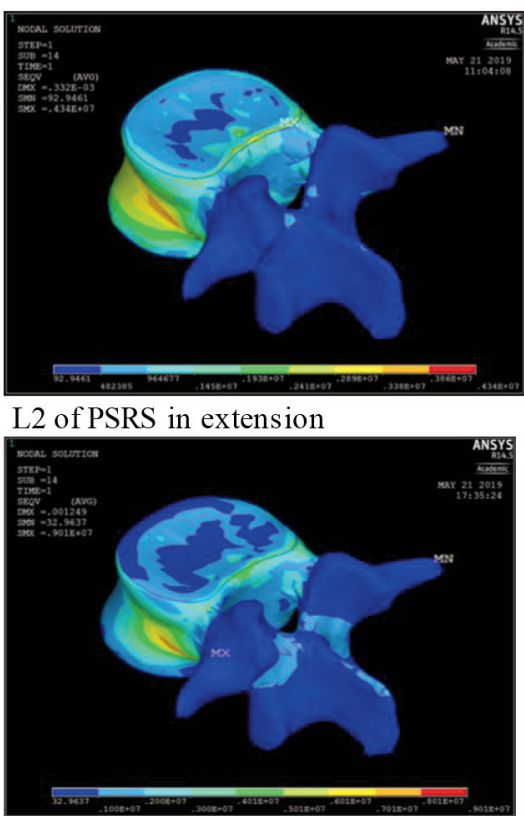

L2 of ISCS in extension

Figure 3. The maximum stress of the middle vertebral body of the two model systems (PSRS and ISCS) of flexion and extension. ISCS, Improved spinal correction system; PSRS, pedicle screw-rod system.

Table V. Stress shielding rate of intervertebral discs and intermediate vertebral bodies in different directions of motion under the same load condition.

\begin{tabular}{|c|c|c|c|c|c|c|}
\hline \multirow[b]{3}{*}{ Direction } & \multicolumn{4}{|c|}{ Stress sheltering rate of disc } & \multirow{2}{*}{\multicolumn{2}{|c|}{$\frac{\text { Stress sheltering rate of centrum }}{\text { L2 }}$}} \\
\hline & \multicolumn{2}{|c|}{$\mathrm{L} 1 / 2$} & \multicolumn{2}{|c|}{$\mathrm{L} 2 / 3$} & & \\
\hline & PSRS & ISCS & PSRS & ISCS & PSRS & ISCS \\
\hline Flexion & 0.38 & 0.13 & 0.28 & 0.19 & 0.31 & 0.14 \\
\hline Extension & 0.68 & 0.34 & 0.64 & 0.36 & 0.80 & 0.59 \\
\hline Left lateral bending & 0.49 & 0.55 & 0.48 & 0.55 & 0.55 & 0.53 \\
\hline Left rotation & 0.64 & 0.66 & 0.54 & 0.55 & 0.21 & 0.14 \\
\hline
\end{tabular}

ISCS, Improved spinal correction system; PSRS, pedicle screw-rod system.

PSRS in flexion, extension, and axial rotation, but it was similar in lateral bending between the two models.

\section{Discussion}

We have recently designed a new improved spinal correction system (ISCS) for surgical therapy of spinal deformity such as scoliosis (1). To exploit the clinical application of the ISCS, we carried out this study to determine its biomechanical characteristics through a comparative finite element analysis of the ISCS and PSRS. We found that ISCS provides a good stability similar to PSRS in coronal plane and horizontal plane, and the advantages of ISCS over PSRS are that it releases a part of motor function in sagittal plane and horizontal plane so as to reduce the stress shielding and stress concentration of fixed vertebral body.

PSRS has been considered as the gold standard for the treatment of scoliosis. The advantages of PSRS include correcting deformity with rigid fixing, higher osseous fusion, and reducing incidence rate of pseudarthrosis. Rigid fixing counters eccentric stress for protecting front and middle columns. Rigid fixing also has higher fusion rate than those with semi-rigid fixing or without fixing. While high stiffness provides immediate stabilization of the scoliosis and reduces 
Table VI. Angular displacement and bending stiffness of L1-L3 in different directions of motion under the same load condition (degree/N•m) in the three models.

\begin{tabular}{|c|c|c|c|c|c|c|}
\hline \multirow[b]{2}{*}{ Loaded state } & \multicolumn{2}{|c|}{ Normal model } & \multicolumn{2}{|c|}{ PSRS } & \multicolumn{2}{|c|}{ ISCS } \\
\hline & $\begin{array}{c}\text { Angular } \\
\text { displacement }\end{array}$ & $\begin{array}{l}\text { Bending } \\
\text { stiffness }\end{array}$ & $\begin{array}{c}\text { Angular } \\
\text { displacement }\end{array}$ & $\begin{array}{l}\text { Bending } \\
\text { stiffness }\end{array}$ & $\begin{array}{c}\text { Angular } \\
\text { displacement }\end{array}$ & $\begin{array}{l}\text { Bending } \\
\text { stiffness }\end{array}$ \\
\hline Flexion & 7.8 & 1.28 & 2.4 & 4.16 & 5 & 2 \\
\hline Extension & 4 & 2.5 & 0.4 & 25 & 2.3 & 4.35 \\
\hline Left lateral bending & 7.7 & 1.30 & 2.3 & 4.35 & 2.4 & 4.17 \\
\hline Left rotation & 2.1 & 4.76 & 1.9 & 5.26 & 2 & 5.00 \\
\hline
\end{tabular}

ISCS, Improved spinal correction system; PSRS, pedicle screw-rod system.

the physiological load of the deformed vertebral body. After fixing by PSRS, the stress is concentrated on the two ends of the vertebral body, pedicle screw and rod, which produces stress shielding effect. The load of the intermediate vertebral body is reduced due to the stress shielding; however, it may cause bone loss and lead to osteoporosis (10-11). Another limitation of PSRS is the shifting of the rotation center of fixed segments to the cephalic and posterior sides. Due to these drawbacks of PSRS, some orthopedics experts and scholars suggest to use biodegradable materials or low elastic modulus materials for internal fixation.

In fact, retaining tiny motion and reducing stress shielding on the basis of rigid fixing are a part of our original purpose and motivational factor to design the ISCS. The transverse process hook of ISCS is made of nickle-titanium memory alloy for medical use whose plasticity is increased at low temperature, and it is convenient for implanting. The transverse process hook grasps the transverse process after thawing. The multi-directional device is convenient for implanting of the rod. Each transverse process hook is connected as a whole by two rods and then scoliosis is corrected three-dimensionally. The transverse process hook can be implanted under direct vision keeping away from the spinal canal. No imaging examination is required during operation thus reducing radiation exposure and operation time. Does the transverse process as a focus point have sufficient bearing capacity? Csernátony and colleagues performed ultimate mechanical tests on the transverse process of adult cadavers with an average age of 67.5 years, and their results show that the transverse process of the cadavers has sufficient capacity to bear the corrective force from internal fixation (15). The CAB system has been verified by biomechanical and animal experiments and has been applied to clinical practice with positive outcome (3). Our ISCS further improved and developed the CAB system.

In the current study, we established a FEM, a PSRS FEM, and an ISCS FEM using the adult lumbar CT data. The same load was applied to the three models for stress test in different directions. (i) In the sagittal plane, we showed that PSRS had
Table VII. Total maximum displacement of L1-L3 segment under $500 \mathrm{~N}$ axial load in the forward bending, backward extension, side bending, and rotation movement under $10 \mathrm{~nm}$ moment $(\mathrm{mm})$.

\begin{tabular}{lclc}
\hline L1 & Normal model & PSRS & ISCS \\
\hline Axial compression & 1.92 & 1.48 & 1.96 \\
Max displacement in flexion & 8.60 & 2.89 & 5.56 \\
Max displacement in extension & 4.75 & 0.497 & 2.86 \\
Max displacement in left lateral & 8.18 & 2.69 & 2.66 \\
bending & 2.73 & 2.13 & 2.57 \\
Max displacement in left rotation & 2.73 & \\
\hline
\end{tabular}

ISCS, Improved spinal correction system; PSRS, pedicle screw-rod system.

smaller angular displacement, maximum displacement, and higher bending stiffness than ISCS in flexion and extension. The main difference between the two models is that screw traverses the three columns of spine through the pedicle providing rigid fixing in PRSR, whereas the transverse process hook of ISCS just fixes the posterior column of spine essentially. In addition, there may be a little movement between hook and transverse process in ISCS. As a part of the interspinous and supraspinous ligament is excised during the implanting of ISCS, the incompleteness of the rear structure is another reason for increasing the ROM of spine in the sagittal plane (12-15). (ii) In the coronal plane, the angular displacement and maximum displacement of L1-3 of the two internal fixings were similar in lateral bending. The fixed point of the hook was bilateral transverse process that ISCS had a longer arm to control the motion of spine in the coronal plane than PSRS. The corrected unit of the two internal fixing systems was both connected by rod whose small elastic modulus and high stiffness restrict the motion of spine in the coronal plane. (iii) In the horizontal plane, the maximum displacement of the model with ISCS was longer than the model with PSRS in axial rotation. Maybe the multidirectional device is inferior to the unidirectional device in controlling axial rotation (16-17). 


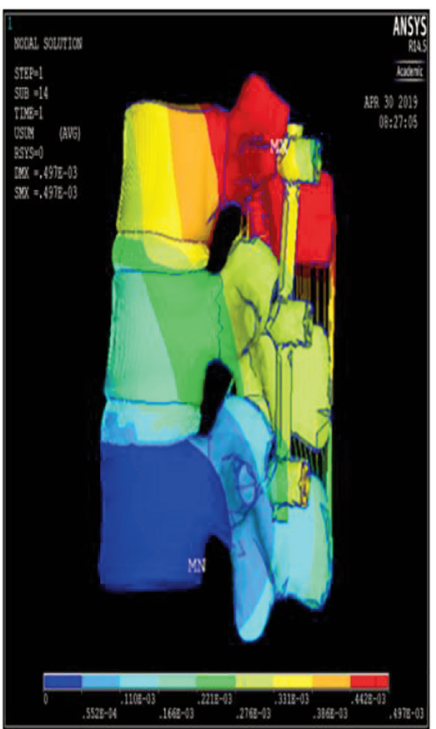

PSRS in extension

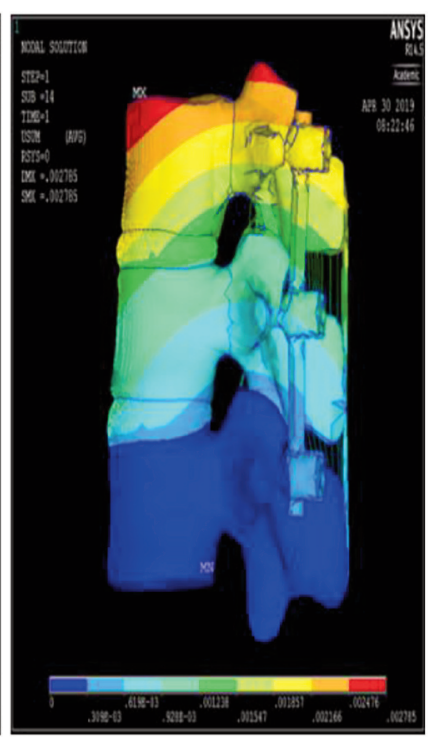

PSRS in flexion

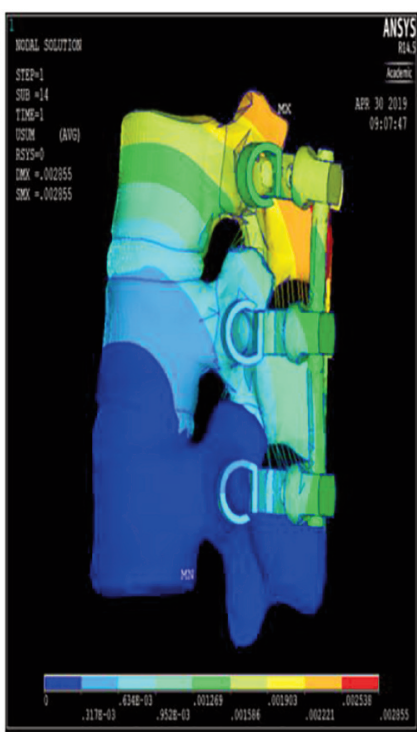

ISCS in extension

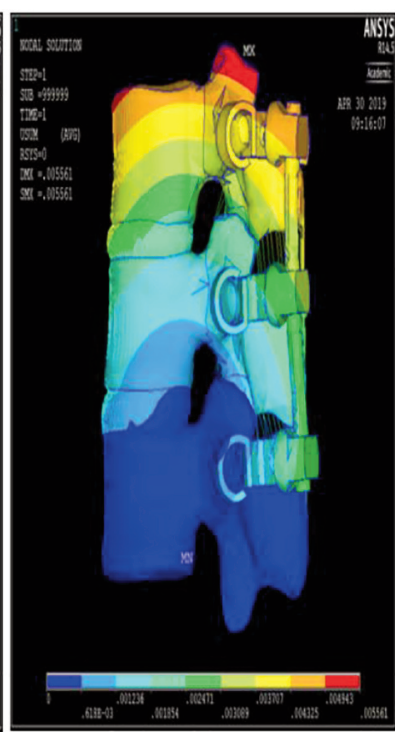

ISCS in flexion

Figure 4. The movement of flexion and extension is the overall maximum displacement (mm). ISCS, Improved spinal correction system; PSRS, pedicle screw-rod system.

As for stress shielding, the stress shielding rate of disc L1/2, L2/3, and centrum L2 from the PSRS model was significantly larger than the ISCS model in flexion and extension, but it was similar in lateral bending and axial rotation (Table V). Therefore, it is concluded that ISCS significantly reduces the stress shielding rate in the sagittal plane compared to PSRS. While in the horizontal plane, due to the rigid fixing of PSRS, stress was transferred from L1 to L3 along the pedicle screws and correction rods, so that the stress of disc L1/2, L2/3, and centrum L2 was smaller in the PSRS model than in the ISCS model.

Adjacent segmental degeneration after spinal internal fixation includes vertebral instability, slippage, osteophyte formation, spinal stenosis, slipped disc, etc. (18-19). Adjacent segmental degeneration is usually associated with a fusion approach, fusion length, mechanical property of internal fixation, and fusion segments. PSRS made the instantaneous center of rotation transfer to proximal segment, which is the major factor causing adjacent segmental degeneration. In the PSRS model, compensatory movement of proximal segments was increased significantly and degeneration in the proximal segments was more conspicuous than the digital ones (20-21). While in the ISCS model, the slight movement between hook and transverse process as well as multi-directional device preserved the proximal segments a little ROM, reduced the stress shielding of intervertebral bone grafts, and retained partial activity function of intervertebral space to delay or reduce adjacent segmental degeneration.
Collectively, our comparative finite element analysis demonstrates that ISCS is effective in internal fixation with advantages in reducing stress shielding and stress concentration. ISCS does not need a pedicle screw or spinal canal, it only needs to expose the transverse process or lamina and allows the surgeon to operate far away from the spinal canal, thus greatly reducing the risk of surgery, reducing complications and improving safety. The system can place the transverse hook under direct vision, which can reduce the radiation exposure and shorten the operation time without imaging examination. The existing research of the research group has also verified the advantages of ISCS compared with traditional methods. Additionally, the device has won a national invention patent and practical patent awards in China. Thus, ISCS is a beneficial supplement for PSRS, and it provides patients with a new option for corrective surgical operation.

The main limitations of the study are that (i) the model was derived from a normal adult rather than from androgen insensitivity syndrome (AIS) patients. Although the main spinal ligaments were added, muscles and tendons were absent, which are different from AIS patients in the clinic. (ii) Patients with AIS often involve with thoracic and lumbar vertebrae. There are differences in transverse process bone mass and anatomical structure between thoracic vertebrae and lumbar vertebrae. (iii) Scoliosis includes lateral curvature in coronal plane, rotation in horizontal plane, and changes of physiological curvature in sagittal plane, the transverse process in AIS is significantly different from the 
normal lumbar vertebra. Therefore, further studies using thoracic vertebra of AIS patients are necessary to elucidate the details of the clinical significance for ISCS.

\section{Conclusion}

The present study was designed to evaluate biomechanical characteristics of ISCS through a comparative finite element analysis of ISCS and PSRS. We demonstrated that (i) ISCS has the advantages of safety and convenience; (ii) ISCS provides similar stability of spine to PSRS in horizontal plane and coronal plane; and (iii) In the sagittal and horizontal planes, ISCS retains partial activity function of the spine to reduce stress shielding and stress concentration, thus reducing adjacent segmental degeneration. These findings support the notion that ISCS provides a new therapeutic option for orthopedic surgery treatment of spinal deformity such as scoliosis and thus warrants further clinical exploration.

\section{Conflicts of Interest}

The Authors declare that they have no conflicts of interest with regard to this study.

\section{Authors' Contributions}

$\mathrm{ZH}$ and MZ conducted the studies, performed the experiments, drafted the article, and are the primary authors of this article. WL, ZL, and LW performed the experiments and analyzed the data. XL and QQL conceived the project, interpreted data, and revised the article. XL obtained the funding and designed the research. All Authors met the authorship criteria and read and approved the final article.

\section{Acknowledgements}

Our sincere thanks are due to all the faculty and staff in the Department of Orthopedics, Southwest Hospital Affiliated to Youjiang Medical College for Nationalities for their help during the study processes. Special gratitude is owed to my (Zubin He) wife Mei Xie for her constant love, encouragement, and strong support to pursue my master's degree in the past three years. This research was supported by grants from BaiSe City Science and Technology Bureau (No. 20150820) and from the Departments of Finance and Science and Technology of Guangxi Province (No. 14123001-24).

\section{References}

1 Lu X: Development and related basic research of a new spinal deformity correction system. Ph.D. Dissertation, Guangxi Med University, 2017 (Adviser: Dr. Jinmin Zhao).

2 Csernátony Z, Goodship A, Szepesi K, Jónás Z, Gáspár L, Benko $\mathrm{K}$ and Lawes $\mathrm{T}$ : A complementary thoracic implant for the surgical correction of the scoliotic curve. A preliminary report. European Journal of Orthopaedic Surgery \& Traumatology 11(2): 85-89, 2019. DOI: 10.1007/BF01682483
3 Csernátony Z, Molnár S, Hunya Z, Manó S and Kiss L: Biomechanical examination of the thoracic spine-the axial rotation moment and vertical loading capacity of the transverse process. J Orthop Res 29(12): 1904-1909, 2011. PMID: 21647957. DOI: $10.1002 /$ jor.21478

4 Csernátony Z, Kiss L, Manó S and Hunya Z: Our experience and early results with a complementary implant for the correction of major thoracic curves. Eur Spine J 22(6): 1286-1291, 2013. PMID: 23392556. DOI: 10.1007/s00586-013-2698-4

5 Goel VK, Monroe BT, Gilbertson LG and Brinckmann P: Interlaminar shear stresses and laminae separation in a disc. Finite element analysis of the L3-L4 motion segment subjected to axial compressive loads. Spine (Phila Pa 1976) 20(6): 689698, 1995. PMID: 7604345.

6 Kim HJ, Chun HJ, Kang KT, Lee HM, Kim HS, Moon ES, Park $\mathrm{JO}$, Hwang $\mathrm{BH}$, Son $\mathrm{JH}$ and Moon SH: A validated finite element analysis of nerve root stress in degenerative lumbar scoliosis. Med Biol Eng Comput 47(6): 599-605, 2009. PMID: 19296142. DOI: 10.1007/s11517-009-0463-y

7 Chen C, Cheng C, Liu C and Lo W: Stress analysis of the disc adjacent to interbody fusion in lumbar spine. Medical Engineering \& Physics 23(7): 485-493, 2019. DOI: 10.1016/ s1350-4533(01)00076-5

8 Shirazi-Adl SA, Shrivastava SC and Ahmed AM: Stress analysis of the lumbar disc-body unit in compression. A threedimensional nonlinear finite element study. Spine (Phila $\mathrm{Pa}$ 1976) 9(2): 120-134, 1984. PMID: 6233710. DOI: 10.1097/ 00007632-198403000-00003

9 Yamamoto I, Panjabi MM, Crisco T and Oxland T: Threedimensional movements of the whole lumbar spine and lumbosacral joint. Spine (Phila Pa 1976) 14(11): 1256-1260, 1989. PMID: 2603060. DOI: 10.1097/00007632-19891100000020

10 Kuklo TR, Potter BK, Polly DW Jr and Lenke LG: Monaxial versus multiaxial thoracic pedicle screws in the correction of adolescent idiopathic scoliosis. Spine (Phila Pa 1976) 30(18): 2113-2120, 2005. PMID: 16166904. DOI: 10.1097/01. brs.0000179260.73267.f4

11 Turner $\mathrm{CH}$ : Three rules for bone adaptation to mechanical stimuli. Bone 23(5): 399-407, 1998. PMID: 9823445. DOI: 10.1016/s8756-3282(98)00118-5

12 Lange T, Schulte TL, Gosheger G, Schulze Boevingloh A, Mayr $\mathrm{R}$ and Schmoelz W: Effects of multilevel posterior ligament dissection after spinal instrumentation on adjacent segment biomechanics as a potential risk factor for proximal junctional kyphosis: a biomechanical study. BMC Musculoskelet Disord 19(1): 57, 2018. PMID: 29444669. DOI: 10.1186/s12891-0181967-0

13 Wu CC, Jin HM, Yan YZ, Chen J, Wang K, Wang JL, Zhang ZJ, $\mathrm{Wu} \mathrm{AM}$ and Wang XY: Biomechanical role of the thoracolumbar ligaments of the posterior ligamentous complex: a finite element study. World Neurosurg 112: e125-e133, 2018. PMID: 29317367. DOI: 10.1016/j.wneu.2017.12.171

$14 \mathrm{Li} \mathrm{Y,} \mathrm{Shen} \mathrm{Z,} \mathrm{Huang} \mathrm{M} \mathrm{and} \mathrm{Wang} \mathrm{X:} \mathrm{Stepwise} \mathrm{resection} \mathrm{of} \mathrm{the}$ posterior ligamentous complex for stability of a thoracolumbar compression fracture: An in vitro biomechanical investigation. Medicine (Baltimore) 96(35): e7873, 2017. PMID: 28858098. DOI: 10.1097/MD.0000000000007873

15 Hartmann F, Janssen C, Böhm S, Hely H, Rommens PM and Gercek E: Biomechanical effect of graded minimal-invasive 
decompression procedures on lumbar spinal stability. Arch Orthop Trauma Surg 132(9): 1233-1239, 2012. PMID: 22592915. DOI: $10.1007 / \mathrm{s} 00402-012-1543-2$

16 Wang X, Aubin CE, Coleman J and Rawlinson J: Correction capability in the 3 anatomic planes of different pedicle screw designs in scoliosis instrumentation. Clin Spine Surg 30(4): E323-E330, 2017. PMID: 28437333. DOI: 10.1097/BSD. 0000000000000082

17 Lin T, Li T, Jiang H, Ma J and Zhou X: Comparing uniplanar and multiaxial pedicle screws in the derotation of apical vertebrae for Lenke $\mathrm{V}$ adolescent idiopathic scoliosis: a casecontrolled study. World Neurosurg 111: e608-e615, 2018. PMID: 29289805. DOI: 10.1016/j.wneu.2017.12.135

18 Ishihara H, Osada R, Kanamori M, Kawaguchi Y, Ohmori K, Kimura T, Matsui H and Tsuji H: Minimum 10-year follow-up study of anterior lumbar interbody fusion for isthmic spondylolisthesis. J Spinal Disord 14(2): 91-99, 2001. PMID: 11285419. DOI: 10.1097/00002517-200104000-00001
19 Miyakoshi N, Abe E, Shimada Y, Okuyama K, Suzuki T and Sato K: Outcome of one-level posterior lumbar interbody fusion for spondylolisthesis and postoperative intervertebral disc degeneration adjacent to the fusion. Spine (Phila Pa 1976) 25(14): 1837-1842, 2000. PMID: 10888954. DOI: 10.1097/ 00007632-200007150-00016

20 Chen S-J: Can hybrid constructs prevent adjacent segments degeneration? Long-term follow-up results. Spine J 19(9): S57S58, 2019. DOI: 10.1016/j.spinee.2019.05.133

21 Rahm MD and Hall BB: Adjacent-segment degeneration after lumbar fusion with instrumentation: a retrospective study. J Spinal Disord 9(5): 392-400, 1996. PMID: 8938607.

Received March 7, 2021

Revised April 16, 2021

Accepted April 19, 2021 